\title{
Influencia de la fuerza muscular isométrica de las extremidades superiores en el estrés oxidativo en niños \\ Influence of handgrip strength in oxidative stress in children
}

\author{
Juan de Dios Benítez-Sillero, Juan Luis Pérez-Navero, Mercedes Gil-Campos \\ Manuel Guillén-del Castillo, Inmaculada Tasset, Isaac Túnez \\ Universidad de Córdoba. España
}

\begin{abstract}
Resumen
El ejercicio físico puede producir estrés oxidativo en el individuo lo que pueden condicionar el riesgo cardiovascular en niños y adolescentes. Este estudio pretende analizar el estrés oxidativo según la fuerza muscular isométrica de las extremidades superiores en la edad pediátrica. Se estudiaron 70 niños sanos con edades entre 10 y 14 años, y se analizaron en saliva los lipoperóxidos (LPO), el glutatión reducido (GSH), la ratio GSH/LPO y la catalasa, como marcadores de estrés oxidativo. La muestra se dividió en dos grupos según una condición física superior o inferior medida a través de dinamometría manual (TKK 5110); se diferenció la serie en sujetos prepuberales y puberales. Se encontraron niveles significativamente inferiores de GSH y GSH/LPO en el grupo de niños con fuerza superior, y en los puberales con la mismas características; estos resultados podrían indicar la existencia de un mayor estrés oxidativo en esta situación. En conclusión, los mayores niveles de fuerza músculo esquelética, medida a través de dinamometría manual, posiblemente pueden estar asociados a un mayor estrés oxidativo en niños púberes con condición física musculoesquelética superior.
\end{abstract}

Palabras clave: estrés oxidativo; fuerza muscular isométrica; dinamometría manual; niños.

\begin{abstract}
Physical exercise can produce oxidative stress, this situation could contribute cardiovascular risk in children and adolescents. The following study tries to evaluate the oxidative stress produced according to the handgrip strength in infancy. 70 healthy male subjects, ages 10 to 14 years, were studied. In the saliva samples, lipoperoxides (LPO), reduced glutathione (GSH), glutathione/lipoperoxides ratio and catalase were analyzed as biomarkers of oxidative stress. Children were divided into two groups according to their handgrip strength (high or low) measured by handgrip strength (TKK 5110). The groups were also divided into prepubertal and pubertal subjects. We found GSH's significantly low levels and of GSH/LPO in pubertal group with high handgrip strength. We could possibly conclude that the higher levels of handgrip strength could be associated with more oxidative stress in pubertal group with high fitness.
\end{abstract}

Key words: oxidative stress; handgrip strength; children.

Correspondencia/correspondence: Juan de Dios Benítez-Sillero

Facultad Ciencias de la Educación. Universidad de Córdoba, Avda. San Alberto Magno s/n. C.P.:14004, Córdoba, España.

e-mail: eo1besij@uco.es 
Benítez-Sillero, J. D.; Pérez-Navero, J.L.; Gil-Campos, M.; Guillén-del Castillo, M.; OTasset, I.; Tunez, I. (2011). Influencia de la fuerza muscular isométrica de las extremidades superiores en el estrés oxidativo en niños. Revista Internacional de Ciencias del Deporte, 22(7), 48-57. http://www.cafyd.com/REVISTA/02205.pdf

\section{Introducción}

$\mathrm{L}$ a condición física (CF) del sujeto está determinada por factores constitucionales, sugiriéndose que al menos el $40 \%$ de las variaciones de la misma pueden ser atribuibles a factores genéticos (Bouchard, 1986; Ruiz et al., 2006). En adultos, una baja CF (especialmente la cardiorrespiratoria, y en menor medida la fuerza muscular) parecen ser predictores potentes de mortalidad, tanto por problemas cardiovasculares como por otras causas, que condicionan la aparición de factores de riesgo (Myers et al., 2002; Ruiz et al., 2006). La fuerza muscular puede ser un predictor de morbimortalidad diferente e independiente (Metter et al., 2002; Hulsmann et al., 2004; Jurca et al., 2005). En estudios longitudinales se ha constatado que el nivel de CF que se posee en la vida adulta, así como la presencia de factores de riesgo cardiovascular conocidos, están en parte condicionados por la forma física que se adquiere durante la infancia y la adolescencia (Janz et al., 2002; Twisk et al., 2002). De hecho, diferentes trabajos publicados han relacionado la CF músculo esquelética o fuerza muscular con factores de riesgo cardiovascular en niños y adolescentes (Twisk et al., 2000; Barnekow-Bergkvist et al., 2001; Hasselstrom et al., 2002, Janz et al., 2002; Ruiz et al., 2009). Por ello, para valorar el riesgo cardiovascular en la edad adulta de forma precoz, hay que tener en cuenta la importancia de obtener una adecuada CF en edades tempranas (Ortega et al., 2005).

Aunque son ampliamente conocidos los beneficios que se derivan del ejercicio físico regular sobre el sistema cardiovascular y la salud, existe evidencia de que durante el ejercicio intenso o extenuante se produce estrés oxidativo (EO) (Ji 1993; Reid 2001). El estrés oxidativo (EO) se caracteriza por un desequilibrio con predominio de la acción de las moléculas de acción oxidante (especies reactivas del oxígeno [EROs]) frente a los mecanismos de defensa antioxidante. Esto da lugar a daño en macromoléculas y muerte celular (Valko et al., 2007). La compensación que tratan de realizar los sistemas antioxidantes del organismo puede ser superada por una sobreproducción de EROs que originan daño oxidativo en el miocardio, músculo esquelético, hígado y otras estructuras orgánicas (Ji, 1993; Reid, 2001). En el sistema de defensa antioxidante tiene una función preponderante el glutatión reducido (GSH), que es una de las principales moléculas antioxidantes presentes en el organismo (Njalsson et al., 2005). Dado que las moléculas antioxidantes son capaces de adaptarse ante una exposición crónica a agentes oxidantes, es de esperar que ante la realización crónica de ejercicio físico (entrenamiento) se produzca una adaptación en respuesta a los agentes oxidantes que produce el ejercicio físico (Jenkins et al., 1988).

Por ello, actualmente, las investigaciones están orientadas a la búsqueda del tipo, intensidad y duración del ejercicio físico ideal y necesario para alcanzar beneficios saludables y evitar la aparición de factores de riesgo de enfermedad.

Los trabajos publicados que relacionan el EO y el ejercicio de fuerza aún son escasos en relación con los que han analizado la relación entre el ejercicio aeróbico y el estrés oxidativo (Hudson et al., 2007). De hecho no hemos encontrado, en la revisión bibliográfica realizada, trabajos que relacionen la fuerza muscular isométrica y el EO durante la infancia o adolescencia. Sólo existen algunos estudios que se centran fundamentalmente en la valoración del efecto de actividades de tipo aeróbico como la natación sobre el estrés oxidativo (Santos Silva et al., 2001; Gonenc et al., 2000; Cavas et al., 2004; Gogugoura et al., 2007; Nikolaidis et al. 2007). En base a lo expuesto, este trabajo pretende establecer si existen diferencias en biomarcadores de estrés oxidativo en saliva (productos de lipoperoxidación, LPO, como especies reactivas de oxigeno; glutatión reducido, GSH, como antioxidante no enzimático y en la actividad de la enzima catalasa, CAT, como antioxidante 
Benítez-Sillero, J. D.; Pérez-Navero, J.L.; Gil-Campos, M.; Guillén-del Castillo, M.; OTasset, I.; Tunez, I. (2011). Influencia de la fuerza muscular isométrica de las extremidades superiores en el estrés oxidativo en niños. Revista Internacional de Ciencias del Deporte, 22(7), 48-57. http://www.cafyd.com/REVISTA/02205.pdf

no enzimático) entre los niños sanos con nivel superior de fuerza muscular isométrica de las extremidades superiores respecto a otros con nivel inferior en situación de reposo.

\section{Material y métodos}

\section{Participantes}

De una muestra total de 100 sujetos de entre 10 y 14 años, se seleccionaron 70 varones caucasianos que cumplieron con todos los criterios de inclusión: estar sano y con peso, talla, índice de masa corporal, frecuencia cardiaca, y presión sistólica y diastólica en valores normales para su edad y sexo \pm una desviación estándar del percentil 50 (p50) (Sobradillo et al., 2004; National High Blood Pressure Education Program, 2004). Se determinó no analizar a un grupo similar de mujeres porque estudios previos en relación al EO no se han encontrado diferencias significativas en función del sexo (Gonenc et al., 2000; Santos Silva et al., 2001; Gogoura et al, 2007; Nikolaidis et al., 2007).

Los criterios de exclusión fueron el presentar patología, periodos de inmovilidad tras enfermedad, lesión, no acudir a alguna de las sesiones, o el consumo de fármacos u otras sustancias (Figura 1). Para mejorar la homogeneidad de la muestra, se tomaron como grupo prepuberal exclusivamente a niños de 10-11 años escolarizados en quinto curso de educación primaria y como grupo puberal niños escolarizados en segundo curso de educación secundaria, descartándose los niños que presentaban un estadio de Tanner diferente al del grupo de edad elegido.

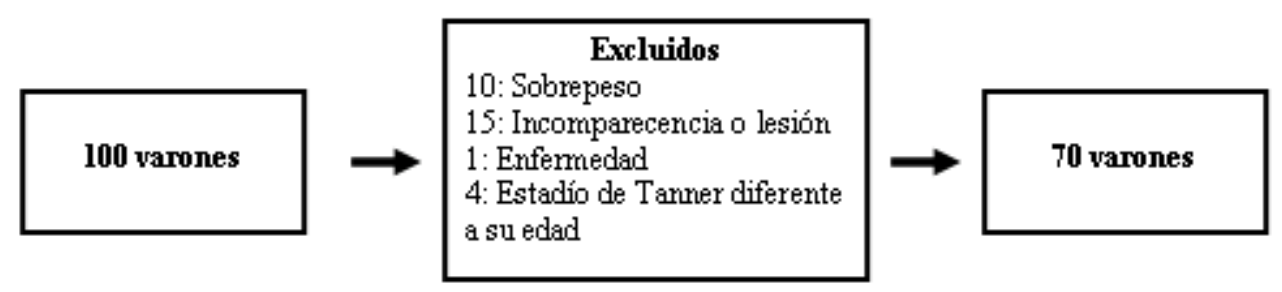

Figura 1. Organigrama de flujo sobre la participación y exclusión de los sujetos en el estudio.

El estudio fue aprobado por el Comité de Bioética e Investigación del Hospital Reina Sofía de Córdoba. Los sujetos fueron incorporados al estudio tras la aceptación voluntaria del menor de su participación y la obtención del consentimiento informado de los padres o del responsable legal.

Valoración antropométrica, hemodinámica y exploración física

Se realizó la anamnesis y la exploración física por sistemas y aparatos, y se determinó el estado puberal (Tanner, 1962), las medidas antropométricas, la frecuencia cardiaca, y la tensión arterial sistólica y diastólica (Tabla 1). Para determinar el peso y la talla respectivamente, se utilizaron una báscula y un estadiómetro (Seca ${ }^{\circledR}$, Hamburg, Germany). La presión sanguínea y la frecuencia cardiaca se determinaron en reposo mediante un monitor de signos vitales 8100T Critikon-Dinamap Vital (Monsey NY).

\section{Evaluación de la condición física musculoesquelética}

Se utilizó el test de dinamometría manual con un dinamómetro digital Takey TKK 5110 (Tokyo, Japan) (rango-100 kg) que mide la fuerza de prensión manual, ya que según Castro 
Benítez-Sillero, J. D.; Pérez-Navero, J.L.; Gil-Campos, M.; Guillén-del Castillo, M.; OTasset, I.; Tunez, I. (2011). Influencia de la fuerza muscular isométrica de las extremidades superiores en el estrés oxidativo en niños. Revista Internacional de Ciencias del Deporte, 22(7), 48-57. http://www.cafyd.com/REVISTA/02205.pdf

et al. (2009) existe una evidencia alta de que este test valora la fuerza muscular de tipo isométrico. Se determinaron las medidas conjuntas, y por separado, con ambas manos (Ortega et al., 2005) para valorar los dos grupos de superior e inferior fuerza muscular isométrica de las extremidades superiores. Para esta clasificación se calcularon los valores medios de la medición para los sujetos de cada rango de edad (10 y 11 años en los prepuberales, y 13 y 14 años para los puberales). Se dividieron en un grupo con superior fuerza muscular isométrica de las extremidades superiores a aquéllos con resultados por encima de la mediana de su edad, y otro grupo con fuerza muscular isométrica de las extremidades superiores a los sujetos con resultados por debajo de la mediana de su edad.

Adicionalmente se valoró la CF cardiorrespiratoria a través del test de Course Navette (Leger, 1984), obteniéndose el $\mathrm{VO}_{2}$ relativo $\left(\mathrm{VO}_{2}\right)$ para cada sujeto (Leger, 1988).

\section{Muestras y análisis bioquímico}

Se recogieron muestras de saliva en situación de reposo evitando la extracción sanguínea, ya que se ha demostrado que existe correlación significativa de los biomarcadores de estrés oxidativo de muestras salivares respecto a valores plasmáticos (Lukash et al., 2002; Yousefzadeh et al., 2006; Bentur et al., 2006).

Las muestras fueron alicuotadas y congeladas a $-70^{\circ} \mathrm{C}$ hasta la realización de las diferentes determinaciones bioquímicas:

GSH: los niveles de GSH $(\mu \mathrm{M})$ fueron determinados utilizando kit de Oxis Internacional (Portland, OR, USA), i.e. GSH-400.

$L P O$ : los niveles de LPO $(\mu \mathrm{M})$ fueron determinados utilizando kit de Oxis Internacional (Portland, OR, USA), i.e. LPO-586.

GSH/LPO: se calculó el cociente GSH/LPO (antioxidante/especie reactiva de oxígeno) como indicador del estrés oxidativo producido (Túnez et al. 2007). Los menores valores del índice indican un mayor estrés oxidativo.

Catalasa: para valorar la actividad de la catalasa (UA/dl) se empleó el método -UV de Aebi (1984).

\section{Análisis estadístico}

Las variables que no se ajustaban a una distribución normal fueron transformadas logarítmicamente. La significación estadística de los cambios entre el grupo de superior e inferior fuerza muscular isométrica fue determinada mediante ANOVA, realizándose a su vez un análisis ANCOVA utilizando la variable IMC como cofactor para las diferencias en las variables de estrés oxidativo. Se valoró el coeficiente de correlación mediante la prueba de Pearson. Los datos se presentan como media \pm desviación estándar. Se consideró como significativa una diferencia con $\mathrm{p}<0,05$. Para el análisis estadístico se utilizó el paquete estadístico SPSS 17 (Chicago, Ill. USA).

\section{Resultados}

En relación con las características antropométricas, el grupo con fuerza isométrica superior no presentó valores significativamente más elevados en peso, talla e índice de masa corporal. Tampoco se encontraron diferencias significativas en las variables hemodinámicas ni en el VO2 relativo (Tabla 1a). Por otra parte, el subgrupo de varones prepuberales con mayor fuerza presentaron mayor peso que el mismo subgrupo con menor fuerza, y en el subgrupo de puberales, los de mayor fuerza presentaron mayor talla (Tabla 1b). 
Benítez-Sillero, J. D.; Pérez-Navero, J.L.; Gil-Campos, M.; Guillén-del Castillo, M.; OTasset, I.; Tunez, I. (2011). Influencia de la fuerza muscular isométrica de las extremidades superiores en el estrés oxidativo en niños. Revista Internacional de Ciencias del Deporte, 22(7), 48-57. http://www.cafyd.com/REVISTA/02205.pdf

Tabla1a: Características demográficas y de condición física para el total de la muestra.

\begin{tabular}{|c|c|c|}
\hline Variables & $\begin{array}{c}\text { Fuerza Superior } \\
\mathrm{n}=35\end{array}$ & $\begin{array}{c}\text { Fuerza Inferior } \\
\mathrm{n}=35\end{array}$ \\
\hline Edad $(\mathrm{años})$ & $12,17 \pm 1,63$ & $12,17 \pm 1,63$ \\
\hline Peso $(\mathrm{Kg})$ & $46,44 \pm 11,49$ & $42,04 \pm 11,25$ \\
\hline Talla $(\mathrm{cm})$ & $154,78 \pm 12,95$ & $149,85 \pm 11,53$ \\
\hline $\mathrm{IMC}\left(\mathrm{Kg} / \mathrm{m}^{2}\right)$ & $19,10 \pm 2,36$ & $18,31 \pm 2,62$ \\
\hline $\mathrm{FC}(\mathrm{Lat} / \mathrm{min})$ & $90,25 \pm 15,64$ & $90,28 \pm 16,72$ \\
\hline $\mathrm{TAS}(\mathrm{mmHg})$ & $118,42 \pm 12,10$ & $117,48 \pm 10,04$ \\
\hline $\mathrm{TAD}(\mathrm{mmHg})$ & $63,11 \pm 10,27$ & $63,05 \pm 12,24$ \\
\hline Fuerza $(\mathrm{Kg})$ & $54,57 \pm 15,74$ & $41,43 \pm 11,91^{\S}$ \\
\hline $\mathrm{VO}_{2}(\mathrm{ml} / \mathrm{kg} / \mathrm{min})$ & $50,65 \pm 4,966$ & $48,20 \pm 5,69$ \\
\hline
\end{tabular}

Tabla1b: Características generales y de condición física en función del estadío puberal.

\begin{tabular}{|l|c|c|l|l|}
\hline & \multicolumn{2}{|c|}{ Prepuberales $\mathrm{n}=38$} & \multicolumn{2}{c|}{ Puberales $\mathrm{n}=32$} \\
\hline \multicolumn{1}{|c|}{ Variables } & $\begin{array}{c}\text { Fuerza Superior } \\
\mathrm{n}=19\end{array}$ & $\begin{array}{c}\text { Fuerza Inferior } \\
\mathrm{n}=19\end{array}$ & $\begin{array}{c}\text { Fuerza Superior } \\
\mathrm{n}=16\end{array}$ & $\begin{array}{c}\text { Fuerza Inferior } \\
\mathrm{n}=16\end{array}$ \\
\hline Edad (años) & $10,74 \pm 0,45$ & $10,74 \pm 0,45$ & $13,88 \pm 0,34$ & $13,8 \pm 0,34$ \\
\hline Peso $(\mathrm{Kg})$ & $37,88 \pm 4,95$ & $34,28 \pm 5,62^{*}$ & $56,59 \pm 8,22$ & $51,25 \pm 9,07$ \\
\hline Talla $(\mathrm{cm})$ & $144,23 \pm 5,06$ & $141,10 \pm 6,30$ & $167,31 \pm 6,41$ & $160,25 \pm 6,45^{+}$ \\
\hline IMC $\left(\mathrm{Kg} / \mathrm{m}^{2}\right)$ & $18,21 \pm 2,29$ & $17,18 \pm 2,26$ & $20,15 \pm 2,04$ & $19,66 \pm 2,42$ \\
\hline FC $(\mathrm{Lat} / \mathrm{min})$ & $91,84 \pm 18,23$ & $92,63 \pm 19,68$ & $88,37 \pm 12,19$ & $87,50 \pm 12,40$ \\
\hline TAS $(\mathrm{mmHg})$ & $114,78 \pm 12,81$ & $115,52 \pm 10,31$ & $122,75 \pm 9,91$ & $119,81 \pm 9,49$ \\
\hline TAD $(\mathrm{mmHg})$ & $65,57 \pm 11,03$ & $66,52 \pm 12,18$ & $60,18 \pm 8,73$ & $58,93 \pm 11,34$ \\
\hline Fuerza $(\mathrm{Kg})$ & $41,28 \pm 5,01$ & $32,05 \pm 3,47^{* *}$ & $70,35 \pm 6,55$ & $52,57 \pm 7,98^{++}$ \\
\hline $\mathrm{VO}_{2}(\mathrm{ml} / \mathrm{kg} / \mathrm{min})$ & $50,02 \pm 5,14$ & $49,50 \pm 5,69$ & $51,40 \pm 4,80$ & $46,66 \pm 5,47$ \\
\hline
\end{tabular}

FC.: Frecuencia Cardiaca; IMC: Índice de masa corporal; TAS: Tensión arterial sistólica; TAD: Tensión arterial diastólica; $\mathrm{VO}_{2}$ : Consumo máximo de oxígeno.

Los datos se transformaron logarítmicamente para las comparaciones, mostrando las medias y SD sin transformar.

$\S ; \mathrm{p}<0,05$ diferencias en el total de la muestra entre grupo de fuerza superior e inferior.

$* ; p<0,05, * * p<0,001$ diferencias en los varones prepuberales entre grupo de fuerza superior e inferior.

$+; \mathrm{p}<0,05,++\mathrm{p}<0,001$ diferencias en los varones puberales entre grupo de fuerza superior e inferior.

Al estudiar las diferentes variables de estrés oxidativo en ambos grupos según la fuerza, se encontraron diferencias significativas entre los valores del GSH e índice GSH/LPO, siendo superiores en el grupo con menor fuerza (Tabla 2). Igualmente, en el subgrupo de niños púberes, los niveles de GSH así como el índice GSH/LPO, fueron significativamente más elevados en el grupo con fuerza inferior. No se encontraron diferencias significativas en los valores de actividad CAT y LPO en ningún grupo o subgrupo (Tabla 3). En el grupo prepuberal, tampoco se encontraron diferencias significativas en ninguno de los valores analizados (Tabla 3). 
Benítez-Sillero, J. D.; Pérez-Navero, J.L.; Gil-Campos, M.; Guillén-del Castillo, M.; OTasset, I.; Tunez, I. (2011). Influencia de la fuerza muscular isométrica de las extremidades superiores en el estrés oxidativo en niños. Revista Internacional de Ciencias del Deporte, 22(7), 48-57. http://www.cafyd.com/REVISTA/02205.pdf

Tabla 2: Variables de estrés oxidativo en función del nivel de fuerza en el total de la muestra.

\begin{tabular}{|l|c|c|c|c|}
\hline \multicolumn{1}{|c|}{$\begin{array}{c}\text { Variables de estrés } \\
\text { oxidativo }\end{array}$} & $\begin{array}{c}\text { Fuerza Superior } \\
\mathrm{n}=35\end{array}$ & $\begin{array}{c}\text { Fuerza Inferior } \\
\mathrm{n}=35\end{array}$ & $\mathrm{p}$ & $\mathrm{p}(\mathrm{IMC})$ \\
\hline $\mathrm{GSH}(\mu \mathrm{mol})$ & $34,70 \pm 26,24$ & $51,06 \pm 39,88^{*}$ & 0,047 & 0,047 \\
\hline $\mathrm{LPO}(\mu \mathrm{mol})$ & $0,30 \pm 0,15$ & $0,290 \pm 0,11$ & 0,628 & 0,707 \\
\hline $\mathrm{CAT}(\mathrm{UA} / \mathrm{L})$ & $1,02 \pm 0,89$ & $1,42 \pm 1,34$ & 0,142 & 0,128 \\
\hline $\mathrm{GSH} / \mathrm{LPO}$ & $120,40 \pm 101,07$ & $177,70 \pm 132,14$ & 0,045 & 0,049 \\
\hline
\end{tabular}

CAT: Catalasa; GSH: Glutation Reducido; LPO: Lipoperóxidos; UA: Unidades de actividad.

Existen diferencias significativas si $\mathrm{p}<0,05$. p: ANOVA. $\mathrm{p}$ (IMC): ANCOVA con IMC como cofactor.

*; $\mathrm{p}<0,05$, diferencias entre grupo de fuerza superior e inferior.

Tabla 3: Variables de estrés oxidativo en función del nivel de fuerza en los grupos prepuberal y puberal.

\begin{tabular}{|c|c|c|c|c|c|c|c|c|}
\hline & \multicolumn{4}{|c|}{ Prepuberales } & \multicolumn{4}{|c|}{ Puberales } \\
\hline $\begin{array}{c}\text { Variables de } \\
\text { Estrés } \\
\text { Oxidativo }\end{array}$ & $\begin{array}{l}\text { Fuerza Superior } \\
\qquad n=19\end{array}$ & $\begin{array}{l}\text { Fuerza Inferior } \\
\qquad \mathrm{n}=19\end{array}$ & $\mathrm{p}$ & $\begin{array}{c}\mathrm{p} \\
\text { (IMC) }\end{array}$ & $\begin{array}{l}\text { Fuerza Superior } \\
n=16\end{array}$ & $\begin{array}{c}\text { Fuerza Inferior } \\
n=16\end{array}$ & $\mathrm{p}$ & $\begin{array}{c}\mathrm{p} \\
\text { (IMC) }\end{array}$ \\
\hline GSH $(\mu \mathrm{mol})$ & $36,39 \pm 29,78$ & $44,22 \pm 45,74$ & 0,535 & 0,508 & $32,70 \pm 22,09$ & $59,18 \pm 31,05^{*}$ & 0,009 & 0,013 \\
\hline LPO $(\mu \mathrm{mol})$ & $0,25 \pm 0,07$ & $0,23 \pm 0,07$ & 0,431 & 0,285 & $0,37 \pm 0,18$ & $0,36 \pm 0,09$ & 0,843 & 0,755 \\
\hline CAT (UA/L) & $0,85 \pm 0,62$ & $1,38 \pm 1,42$ & 0,147 & 0,133 & $1,22 \pm 1,11$ & $1,48 \pm 1,27$ & 0,555 & 0,580 \\
\hline GSH/LPO & $145,20 \pm 123,17$ & $186,88 \pm 166,47$ & 0,386 & 0,320 & $90,54 \pm 56,98$ & $166,80 \pm 78,0^{*}$ & 0,004 & 0,005 \\
\hline
\end{tabular}

CAT: Catalasa; GSH: Glutation Reducido; LPO: Lipoperóxidos; UA: Unidades de actividad.

Existen diferencias significativas si $\mathrm{p}<0,05$. $\mathrm{p}$ : ANOVA. $\mathrm{p}$ (IMC): ANCOVA con IMC como cofactor.

*; $\mathrm{p}<0,05$, diferencias entre grupo de fuerza superior e inferior.

Al realizar correlaciones con el total de niños estudiados, el IMC se correlacionó positivamente con la fuerza $(\mathrm{r}=0,48 ; \mathrm{p}=0,000)$. Asimismo, la fuerza se correlacionó significativamente con los LPO $(\mathrm{r}=0,40 \mathrm{p}=0,001)$ y con el índice GSH/LPO de forma negativa $(\mathrm{r}=-0,29 \mathrm{p}=0,013)$. Igualmente, en el grupo de púberes la fuerza se correlacionó negativamente con el índice GSH/LPO ( $\mathrm{r}=-0,38 \mathrm{p}=0,032)$; en el grupo de prepúberes no hubo correlaciones significativas. 
Benítez-Sillero, J. D.; Pérez-Navero, J.L.; Gil-Campos, M.; Guillén-del Castillo, M.; OTasset, I.; Tunez, I. (2011). Influencia de la fuerza muscular isométrica de las extremidades superiores en el estrés oxidativo en niños. Revista Internacional de Ciencias del Deporte, 22(7), 48-57. http://www.cafyd.com/REVISTA/02205.pdf

\section{Discusión}

En el presente estudio se han evaluado los biomarcadores de estrés oxidativo en función del nivel de fuerza muscular isométrica de las extremidades superiores, poniendo de manifiesto que los niños en el grupo puberal, muestran mayores niveles de estrés oxidativo cuando tienen un nivel superior de fuerza muscular isométrica de las extremidades superiores, mostrando unos menores niveles de GSH y del índice GSH/LPO. El GSH desempeña numerosas e importantes funciones metabólicas (Powers et al., 2008), entre ellas actuar como antioxidante en la protección celular contra los radicales libres, peróxidos y otros compuestos tóxicos. Por ello, el descenso del GSH es considerado un marcador de estrés oxidativo por diversos autores (Subudhi et al., 2003: Bloomer et al., 2004).

En algunos estudios se ha encontrado que niños y adolescentes obesos pueden presentar menores niveles de GSH (Codoñer-Franch et al., 2010) y catalasa, así como mayores productos de peroxidación lipídica (malondialheido (MDA)) (Zhu et al., 2006). En el presente trabajo, no parece haber relación con el IMC, ya que tras hacer el ajuste con este factor, los resultados permanecen similares y continúan las diferencias significativas sin que haya además, correlaciones entre el IMC y los parámetros de estrés oxidativo evaluados. No obstante, una de las limitaciones del estudio, es el no poder valorar exactamente la fuerza en relación a la masa muscular y al tipo de fibra muscular de las extremidades superiores, que no se pudieron evaluar.

Son pocos los estudios donde se analiza la relación entre el ejercicio físico de fuerza y el estrés oxidativo (Ortenblad et al., 1997; McBride et al., 1998; Child et al., 1998; Ramel et al., 2004; Hudson, 2007; Kara et al., 2010. Sin embargo, diferentes estudios en niños (Santos Silva et al., 2001; Cavas et al., 2004; Gougoura et al. 2007) han observado niveles inferiores de GSH, así como de otros antioxidantes (Alshammari et al., 2010), en aquéllos niños sometidos a entrenamiento o actividad física frente a otros que no estaban sometidos a esta actividad, lo cual debemos considerar con cautela ya que en dichos estudios se refiere al nivel de actividad física mientras que nuestro estudio al nivel de fuerza muscular isométrica de las extremidades superiores. En la revisión bibliográfica efectuada hay un trabajo que estudia la fuerza muscular isométrica de las extremidades superiores y el estrés oxidativo en mujeres postmenopaúsicas (Morimoto et al., 2008), sin encontrar información en niños y adolescentes. Recientemente, se ha publicado un estudio en adolescentes en los que no encuentran diferencias en los niveles del antioxidante GSH en los sujetos que han seguido un entrenamiento de fuerza $v s$ los no entrenados (Kara et al, 2010).

Adicionalmente, han sido detectados aumentos en los niveles plasmáticos de MDA, después del ejercicio tanto de resistencia aeróbica como de fuerza-resistencia (McBride et al., 1998; Child et al., 1998). También se ha observado aumento de la concentración de MDA en sujetos no entrenados comparados con entrenados sometidos a ejercicio de fuerza-resistencia (Ramel et al., 2004). Estos resultados parecen indicar que la práctica regular de ejercicio de fuerza-resistencia se asociada a un menor grado de peroxidación lipídica durante este tipo de esfuerzos (Ramel et al., 2004). Sin embargo, Ortenblad et al. (1997) no encontraron diferencias en estos biomarcadores en ejercicios de fuerza-resistencia, atribuida a un aumento de enzimas antioxidantes como la superóxido dismutasa, la glutatión reductasa y la glutatión peroxidasa. Junto a esto, el estudio de Morimoto et al. (2008) no pone de manifiesto la existencia de una relación entre el estrés oxidativo y el estrés físico que se lleva a cabo en mujeres pre y postmenopaúsicas a través del test de dinamometría manual, seguramente debido a la poca intensidad del test (30\% de la contracción máxima). 
Benítez-Sillero, J. D.; Pérez-Navero, J.L.; Gil-Campos, M.; Guillén-del Castillo, M.; OTasset, I.; Tunez, I. (2011). Influencia de la fuerza muscular isométrica de las extremidades superiores en el estrés oxidativo en niños. Revista Internacional de Ciencias del Deporte, 22(7), 48-57. http://www.cafyd.com/REVISTA/02205.pdf

Los resultados de niños púberes en este estudio muestran que el grupo con mayor fuerza tiene menores niveles de antioxidante GSH e índice GSH/LPO, lo que puede indicar un mayor EO en estos sujetos. Estos datos, parecen estar en la línea de resultados previos publicados por nuestro grupo, que muestran como el EO es mayor en niños puberales respecto a los prepuberales en situación basal (Pérez-Navero et al., 2009). No se conoce aún el mecanismo de actuación de los marcadores de oxidación y antioxidación en las diferentes etapas de la pubertad, pero estas diferencias podrían deberse a diversos factores, como la actuación de diferentes hormonas que actúan en el proceso biológico de la pubertad, el acúmulo de productos de lipoperoxidación por el fenómeno de envejecimiento (Pérez-Navero et al., 2009), o la mayor masa muscular en los niños púberes.

\section{Conclusiones}

En base a lo expuesto, podemos concluir que niveles mayores de fuerza muscular isométrica de las extremidades superiores están asociados a un mayor estrés oxidativo en niños púberes. Sin embargo, son necesarios más estudios en diferentes grupos de edad y sometidos a diferentes intensidades y tipos de ejercicio físico, para establecer los mecanismos implicados.

\section{Referencias}

Aebi, H. (1984). Catalase in vitro. Methods Enzymol, 34, 479-500.

Alshammari, E.; Shafi, S.; Nurmi-Lawton, J.; Taylor, A.; Lanham-New, S.; \& Ferns, G. (2010). Altered antioxidant and trace-element status in adolescent female gymnasts. Int J Sport Nutr Exerc Metab, 20(4), 291-298.

Bentur, L.; Mansour, Y; Brik, R.; et al. (2006) Salivary oxidative stress in children during acute asthmatic attack and during remission. Respir Med, 100, 1195-1201.

Bouchard, C. (1986). Genetics of aerobic power and capacity. In Sports and Human Genetics. Champaign, IL: Human Kinetics.

Barnekow-Bergkvist, M.; Hedberg, G.; Janlert, U.; \& Jansson, E. (2001). Adolescent determinants of cardiovascular risk factors in adult men and women. Scand J Public Health, 29(3), 208-217.

Cavas, L.; \& Tarhan, L. (2004). Effects of vitamin-mineral supplementation on cardiac marker and radical scavenging enzymes, and MDA levels in young swimmers. Int $J$ Sport Nutr Exerc Metab, 14(2), 133-146.

Castro-Piñero, J.; Artero, E.G.; España-Romero, V.; Ortega, F.B.; Sjöström, M.; Suni, J., et al. (2010). Criterion-related validity of field-based fitness tests in youth: A systematic review. Br J Sports, 44(13), 934-043.

Child, R.; Wilkinson, D.M.; Brown, S.; Fallowfield, J.L.; \& Donnelly, A. (1998). Elevated serum antioxidant capacity and plasma malondialdehyde concentration in response to a simulated half-marathon run. Med Sci Sports Exerc, 30, 1603-1607

Codoñer-Franch, P.; Boix-García, L.; Simó-Jordá, R.; Del Castillo-Villaescusa, C.; MasetMaldonado, J.; \& Valls-Bellés, V. (2010). Is obesity associated with oxidative stress in children? Int J Pediatr Obes, 5(1), 56-63.

Gonenc, S.; Acikgoz, O.; Semin, I.; \& Ozgonul, H. (2000). The effect of moderate swimming exercise on antioxidant enzymes and lipid peroxidation levels in children. Indian J Physiol Pharmaco, 44(3), 340-344. 
Benítez-Sillero, J. D.; Pérez-Navero, J.L.; Gil-Campos, M.; Guillén-del Castillo, M.; OTasset, I.; Tunez, I. (2011). Influencia de la fuerza muscular isométrica de las extremidades superiores en el estrés oxidativo en niños. Revista Internacional de Ciencias del Deporte, 22(7), 48-57. http://www.cafyd.com/REVISTA/02205.pdf

Gougoura, S.; Nikolaidis, M.G.; Kostaropoulos, I.A.; Jamurtas, A.Z.; Koukoulis, G.; \& Kouretas, D. (2007). Increased oxidative stress indices in the blood of child swimmers. Eur J Appl Physiol, 100(2), 235-239.

Hasselstrom, H.; Hansen, S.E.; Froberg, K.; \& Andersen, L.B. (2002). Physical fitness and physical activity during adolescence as predictors of cardiovascular disease risk in young adulthood. Danish Youth and Sports Study. An eight-year follow-up study. Int J Sports Med, 23 Suppl 1, S27-31.

Hudson, M.B.; Hosick, P.A. McCaulley, G.O.; Schrieber, L.; Wrieden, J. McAnulty, S.R. Triplett, N.T.; McBride, J.M.; \& Quindry, J.C. (2008). The effect of resistance exercise on humoral markers of oxidative stress. Med Sci Sports Exerc, 40(3), 542-548.

Janz, K.F.; Dawson, J.D.; \& Mahoney, L.T. (2002). Increases in physical fitness during childhood improve cardiovascular health during adolescence: the Muscatine Study. Int J Sports Med, 23,15-21.

Ji, LL.(1993) Antioxidant enzyme response to exercise and aging. Med Sci Sports Exerc, $25,225-231$.

Kara, E.; Gunay, M.; Cicioglu, I.; Ozal, M.; Kilic, M.; Mogulkoc, R.; \& Baltaci, AK. (2010) Effect of zinc supplementation on antioxidant activity in young wrestlers. Biol Trace Elem Res, (1),55-63.

Léger, L.; Lambert, A.; Goulet, A.; Rowan, C.; \& Dinelle, Y. (1984). Aerobic capacity of 6 to 17-year-old Quebecois--20 meter shuttle run test with 1 minute stages. Can J Appl Sport Sci, 9, 64-69.

Léger, L.A.; Mercier, D. Gadoury, C.; \& Lambert, J. (1988). The multistage 20 metre shuttle run test for aerobic fitness. J Sports Sci, 6, 93- 101.

Lukash, A.I.; Zaika, V.G.; Kucherenko, A.O.; et al. (2002) Free radical processes and antioxidant system in depression and treatment efficiency. Zh Nevrol Psikhiatr Im S S Korsakova, 102:41-44.

McBride, J.M.; Kraemer, W.J. Triplett- McBride, T.; \& Sebastianelli, W. (1998). Effect of resistance exercise on free radical production. Med Sci Sports Exerc, 30, 67-72

Morimoto, K.; Morikawa, M.; Kimura, H.; Ishii, N.; Takamata, A.; Hara, Y.; Uji, M.; \& Yoshida, K. (2008). Mental stress induces sustained elevation of blood pressure and lipid peroxidation in postmenopausal women. Life Sci, 2;82(1-2), 99-107.

Myers, J.; Prakash, M.; Froelicher, V.; Do, D.; Partington, S.; \& Atwood, J.E. (2002). Exercise capacity and mortality among men referred for exercise testing. $N$ Engl $J$ Med, 346, 793-801.

National High Blood Pressure Education Program Working Group on High Blood Pressure in Children and Adolescents. (2004) The Fourth Report on the Diagnosis, Evaluation, and Treatment of High Blood Pressure in Children and Adolescents. Pediatrics, 114(2)

Nikolaidis, M.G.; Kyparos, A.; Hadziioannou, M.; Panou, N.; Samaras, L.; Jamurtas, A.Z.; \& Kouretas, D. (2007). Acute exercise markedly increases blood oxidative stress in boys and girls. Appl Physiol Nutr Metab, 32(2), 197-205.

Ortenblad, N.; Madsen, K.; \& Djurhuus, M.S. (1997). Antioxidant status and lipid peroxidation after short -term maximal exercise in trained and untrained humans. Am J Physiol, 272: R1258-1263.

Ortega, F.B.; Ruiz, J.R.; Castillo, M.J.; González-Gross, M.; Warnberg, J.; \& Gutiérrez A. (2005). Low Level of Physical Fitness in Spanish Adolescents. Relevance for Future Cardiovascular Health (AVENA Study). Rev Esp Cardiol, 58(8), 889-909. 
Benítez-Sillero, J. D.; Pérez-Navero, J.L.; Gil-Campos, M.; Guillén-del Castillo, M.; OTasset, I.; Tunez, I. (2011). Influencia de la fuerza muscular isométrica de las extremidades superiores en el estrés oxidativo en niños. Revista Internacional de Ciencias del Deporte, 22(7), 48-57. http://www.cafyd.com/REVISTA/02205.pdf

Pérez-Navero, J.L.; Benítez-Sillero, J.D.; Gil-Campos, M.; Guillénn-del Castillo, M.; Tasset, I.; \& Túnez, I. (2009). Cambios en biomarcadores del estrés oxidativo inducidos por la pubertad. An Pediatr (Barc), 70(5):424-428

Powers, S.K.; Jackson, M.J. (2008). Exercise-induced oxidative stress: cellular mechanisms and impact on muscle force production. Physiol Rev, 88(4), 1243-1276.

Ramel, A.; Wagner, K.H.; \& Elmadfa, I. (2004). Plasma antioxidants and lipid oxidation after submaximal resistance exercise in men. Eur J Nutr, 43, 2-6

Reid, M.B. (2001). Nitric oxide, reactive oxygen species, and skeletal muscle contraction. Med Sci Sports Exerc, 33(3), 371-376.

Ruiz, J.R.; Ortega, F.B.; Gutiérrez, A.; Meusel, D.; Sjöström, M.; \& Castillo, M.J. (2006). Health-related fitness assessment in childhood and adolescence; a European approach based on the AVENA, EYHS and HELENA studies. J Public Health, 14, 269277.

Ruiz, J.R.; Castro-Piñero, J.; Artero, E.G.; Ortega, F.B.; Sjöström, M. Suni, J.; \& Castillo, M.J. (2009). Predictive validity of health-related fitness in youth: a systematic review. Br J Sports Med, 43(12), 909-923.

Santos-Silva, A.; Rebelo, M.I.; Castro, E.M.; Belo, L. Guerra, A.; Rego, C. \& Quintanilha, A. (2001). Leukocyte activation, erythrocyte damage, lipid profile and oxidative stress imposed by high competition physical exercise in adolescents. Clin Chim Acta, 306(1-2), 119-126.

Sobradillo, B.; Aguirre, A.; Arsti, U.; Bilbao, A.; Fernández-Ramos, C.; Lizárraga, A.; Lorenzo, H. et al. (2004). Curvas y Tablas de crecimiento (estudios longitudinal y transversal). Instituto de Investigación sobre Crecimiento y Desarrollo. Bilbao; Fundación Faustino Orbegozo Eizaguirre.

Tanner, J.M. (1962). Growth at adolescent. Oxford: Blackwell.

Túnez, I.; Muñoz, M.C.; Montilla, P.; \& Tasset, I. (2007). The Effect of 3-Nitropropionic acid on nitrative and oxidative stress in N1E-115 Neuroblastoma Cells. En: Webster LR. editor. Neurotoxicity Syndromes. New York: Nova Science Publisher Inc. 125-140

Twisk, J.W.; Kemper, H.C.; \& Van Mechelen, W. (2000). Tracking of activity and fitness and the relationship with cardiovascular disease risk factors. Med Sci Sports Exerc, 32(8), 1455-1461.

Twisk, J.W.; Kemper, H.C.; \& Van Mechelen, W. (2002). Prediction of cardiovascular disease risk factors later in life by physical activity and physical fitness in youth: general comments and conclusions. Int J Sports Med, 23, 44-49.

Yousefzadeh, G.; Larijani, B.; Mohammadirad, A. et al. (2006). Determination of oxidative stress status and concentration of TGF-beta 1 in the blood and saliva of osteoporotic subjects. Ann N Y Acad Sci, 1091, 142-150. 